Evidence for the belief in live lineup superiority

\author{
Heather L. Price ${ }^{1}$, Madison B. Harvey ${ }^{2}$, Shelbie F. Anderson ${ }^{2}$, Leah Chadwick ${ }^{1}$, \\ $\&$ \\ Ryan J. Fitzgerald ${ }^{3}$ \\ ${ }^{1}$ Thompson Rivers University, Canada \\ ${ }^{2}$ Simon Fraser University, Canada \\ ${ }^{3}$ University of Portsmouth, United Kingdom
}

This is a post-peer-review, pre-copyedit version of an article published in Journal of Police and Criminal Psychology. The final authenticated version is available online at: https://doi.org/10.1007/s11896-018-9305-x 


\begin{abstract}
There are substantive differences in international preferences in the medium through which eyewitness identification lineups are administered, but these jurisdictional preferences are not based in evidence. Regardless of whether one medium produces greater identification accuracy, it is the perception of evidence strength by triers of fact that determines its influence in judicial proceedings. Yet, we know little about how lineup mediums are perceived by potential triers of fact. Four-hundred and six undergraduate students viewed a video interview of an eyewitness describing an identification that took place with one of three different lineup mediums (live, video, photo) to compare their relative persuasiveness. Participants also directly compared mediums. There was a clear preference for evidence elicited from live lineups in direct comparisons, but not in the experimental conditions. Live lineup superiority beliefs exist in policy and, these data show, in the beliefs of potential witnesses and triers of fact when various lineup mediums are directly compared.
\end{abstract}

Keywords: lineup medium; eyewitness identification; live superiority hypothesis 


\section{Evidence for the belief in live lineup superiority}

There is substantial international variation in the medium through which eyewitness lineup identifications are presented. Due to practical challenges posed by the construction, organization, and administration of live lineups (Valentine \& Heaton, 1999), many jurisdictions have adopted nonlive alternatives. Photo lineups are easy to construct, portable, and simple to administer. Video lineups share many of the advantages of photo lineups and also provide dynamic views of the lineup members. These practical advantages, however, are up against the intuitive appeal of live lineups, which pass the 'eyeball' test of appearing on the surface to provide the most information to a potential witness. Nevertheless, experimental research has yet to demonstrate that live lineups are any better than nonlive alternatives (Brewer \& Palmer, 2010; Clark, Moreland, \& Rush, 2015; Cutler, Berman, Penrod, \& Fisher, 1994; Fitzgerald, Price, \& Valentine, 2018).

Despite the absence of empirical evidence, witnesses and triers of fact may intuit that live lineups would produce the most accurate identification evidence. Fitzgerald et al. (2018) reviewed eyewitness identification practices in five countries (Australia, Canada, England and Wales, South Africa, United States) and found they all either once had or still have policies consistent with a live superiority hypothesis. Whether a potential juror or judge similarly believes in live superiority is a critical question because, even if a host of practical challenges accompany live lineups, it is the perception of the strength of the evidence that will determine its persuasiveness and influence in judicial proceedings. In the present study, we examined perceptions of evidence elicited with one of three different lineup mediums (live, video, photo) in an attempt to better understand how potential triers of fact perceive such evidence. We studied this question from two perspectives: 1) we examined perceptions of lineup mediums in a 
between-subjects vignette study to explore potential influences within the context of a witness' testimony, and 2) we directly asked participants to compare lineup mediums and indicate their preference.

\section{Identification Outcomes by Lineup Medium in the Field and in the Lab}

Although live lineups were once the preferred medium in England and Wales, Home Office guidance now indicates a preference for video lineups. In addition to the practical and economic benefits of presenting reusable video recordings of the lineup members, the policy change was precipitated by empirical research involving lineups conducted in the field. The first relevant data were reported by Valentine and Heaton (1999), who showed that video lineups were fairer to suspects compared with live lineups (see also Valentine, Harris, Piera, \& Darling, 2003). The second set of field data were archival records of identifications from live and video lineups, collected by the Video Identification Parade Electronic Recording (VIPER) unit of the West Yorkshire Police force (Pike, Brace, \& Kynan, 2002). In the archival data, the suspect identification rate for video lineups was significantly higher than the rate for live lineups. There are several caveats to these field data: the difference in suspect identifications was small (39\% vs $35 \%$, respectively); the ground truth of the suspect's guilt was unknown; and the allocation of witnesses to a lineup medium was not random because at that time video lineups were only permitted when live lineups were not practical. Nevertheless, given the relative ease of organizing and administering video lineups, and the absence of any indication that video lineups reduce suspect identifications, Pike et al. (2002) recommended abandoning the preference for live lineups and giving video lineups equivalent status. Police forces in England and Wales started to transition away from live lineups in 2003, and they now use video presentation in virtually every lineup case (Valentine, Hughes, \& Munro, 2009). 
Consistent with the archival field research, live lineups have not outperformed photo or video lineups in laboratory experiments. In their review of 11 experimental comparisons between live and nonlive lineups, all of which were laboratory experiments, Fitzgerald et al. (2018) found that none provided strong support for the live superiority hypothesis and one set of experiments was actually suggestive of live inferiority compared to photos (Dent \& Stephenson, 1979).

Fitzgerald et al. also reported that participants seemed more reluctant to choose from live relative to nonlive lineups (i.e., more conservative responding; Cutler \& Fisher, 1990; Dent \& Stephenson, 1979; Kerstholt, Koster, \& van Amelsvoort, 2004; Peters, 1991).

\section{Theoretical and Applied Consequences of a Live Superiority Hypothesis}

A widespread belief in live superiority could explain the conservative responding associated with live lineups. If witnesses believe that they would be best able to identify someone at a live lineup, they may expect that the culprit, if present, would elicit a stronger experience of recognition than would be expected at a nonlive lineup. This difference in expectations could lead participants to increase their threshold for identifying a lineup member from a live lineup relative to a threshold they would have applied for a photo or video lineup. To explore this explanation of the conservative response bias in live lineups, it must first be established that participants in lineup experiments (typically undergraduate students) believe that live lineups are superior to nonlive lineups.

Empirical evidence of the live superiority hypothesis would also have policy implications. Australia and South Africa have policies that indicate a preference for live lineups (Australian Law Reform Commission, 2006; Rust \& Tredoux, 1998; $S$ v. Moti, 1998). Even in England and Wales, where video lineups are now standard practice, live lineups are preferred over photo lineups in policy (Home Office, 2017). But technological advances have led to 
significant increases in the quality of photo and video images since the origin of these policies, and the preference for live lineups may reflect more of a continuation of tradition than a contemporary belief that photo and video lineups are inferior. Although a belief in the superiority of live lineups is evident in eyewitness identification policies throughout the world, empirical data are needed to assess whether the live superiority hypothesis is pervasive in the digital age.

\section{The Present Study}

In the present research, participants watched a video interview of an eyewitness describing an identification from a live, photo, or video lineup. After providing a judgment of guilt for the case, participants were queried about which of the three lineup mediums would be most likely to produce a correct identification decision. We hypothesized that we would observe evidence for a live superiority belief, which would be supported both by higher guilt ratings when the witness described an identification from a live lineup (vs. nonlive lineups) and by a preference for live lineups in the medium comparison queries.

We also manipulated viewing conditions (lighting: well-lit, dark) and the certainty of the eyewitness $(70 \%, 90 \%$ confident $)$ to assess participants' sensitivity within each medium to the strength of identification evidence. Consistent with prior research, we anticipated that witnesses would place greater value on identifications made with higher confidence (e.g., Brewer \& Burke, 2002). With little direct guidance related to lighting conditions during encoding (but see Yarmey, 1986), we nonetheless hypothesized that witnessing the suspect in good lighting would be perceived as 'good viewing conditions' and thus, a resulting identification would be evaluated more favorably than witnessing the suspect in poor lighting. We also anticipated that lineup medium may interact with evidence strength (confidence, lighting) such that a witness would be more likely to forgive low confidence or poor lighting if the identification was obtained with a 
preferred lineup medium. That is, we anticipated that participants would treat lineup medium as contributing to evidence strength, which would be balanced with other evidence strength factors (lighting and confidence).

\section{Method}

\section{Participants}

Undergraduate students $(\mathrm{N}=406 ; M$ age $=20.61$ years; $65 \%$ female $)$ at two universities in Canada participated individually or in small groups of up to 10 for course credit. Participants were quasi-randomly assigned to conditions, with the restriction of equal distribution across conditions. This study was a 3 (Lineup medium: photo, live, video) x 2 (Witness confidence: $70 \%, 90 \%)$ x 2 (Viewing conditions: light, dark) between-subjects design.

\section{Materials and Procedure}

Eyewitness testimony videos. Participants watched a video (approximately 70 seconds in duration) featuring two actors. A 28-year-old male 'witness' answered questions posed by a 'prosecutor' about a witnessed assault and robbery. The witness described the crime and then answered questions about his lineup identification of the accused. In addition to a standard crime description, the witness discussed the medium in which the lineup was presented (photo, live, or video), how confident he was in his identification $(70 \%$ or $90 \%)$, and the viewing conditions at the scene of the crime (well-lit or dark). Thus, 12 different versions of the video were presented. To manipulate lineup medium, the witness briefly described each medium in the following manner:

1) Photo: “...showed me a bunch of pictures of guys to look at one at a time, you know like, head and shoulders shots..." 
2) Video: “...showed me a bunch of videos of guys to look at one at a time, you know, like videos of just their heads and shoulders, but they turn so I can see their faces...."

3) Live: “...showed me a bunch of guys one at a time behind a one-way mirror so I could look at them without them seeing me..."

To manipulate viewing conditions, the witness described the lighting in one of two ways:

1) "...there was a streetlight he ran under, so I got a pretty good look at him."

2) “...it was pretty dark, so I didn't really see him all that well."

Finally, the witness described his confidence as either:

1) "...I told the cops that I was pretty sure it was him. Like, 70\% sure."

2) "...I told the cops that I was pretty sure it was him. Like, 90\% sure."

We focused our investigation on sequential lineups because sequential presentation is used for all three mediums in practice, whereas simultaneous presentation is only used for photo and live mediums.

Video evaluations. After viewing the video, participants rated the likelihood that the accused committed the crime from $0 \%$ (extremely unlikely) to $100 \%$ (extremely likely) and made a categorical verdict decision (guilty/not guilty). Participants were then asked to provide narrative descriptions of any concerns they had with the witness' identification ("Do you have any concerns about the witness' identification?") and factors that may have increased their confidence in the accuracy of the identification ("What might have made you more sure about the accuracy of the witness 'identification?"). Three questions were then posed to assess participants' attention to the manipulations in the video: Did the witness get a good look at the suspect? How confident was the witness? What did the police give the witness to make the identification (live, photo, video)? 
General lineup impressions. Following questions about the experimental manipulations, participants responded to questions querying their opinions regarding the reliability of a variety of lineup mediums in general. They were asked to indicate which lineup medium (simultaneous or sequential: live, photo, or video) they believed would be the best procedure to accurately identify a perpetrator, should they personally have witnessed a crime. They were then asked to explain their choice. Next, they were asked to rate from 0 (not at all confident) to 100 (extremely confident) how confident they would feel making an identification with each of the six lineup mediums listed above. Finally, participants indicated which of the six mediums would be the best procedure to ensure that someone else could accurately identify a perpetrator.

Procedure. Participants arrived at the lab and were informed that they would watch a short video and answer some questions. After viewing one of the 12 videos, participants provided demographic information and then were asked to take on the role of a juror while completing the questionnaires (approximately 10 minutes). Participants were then debriefed and thanked.

\section{Coding}

Open-ended responses were reviewed by two coders and the first author to generate categories of responses. Next, the two coders independently coded the complete sample to obtain intercoder agreement. Agreement for each question ranged from $83 \%$ to $86 \%$.

\section{Results}

Three attention-check questions were posed during the experiment. Participants who failed to correctly respond to all three questions ( $n=10$ ) were excluded from analyses. Thus, the total sample collected of 416 was reduced to 406 for analyses. 
Suspect guilt. Participants' ratings of the likelihood of suspect guilt (from 0\%- extremely unlikely to $100 \%$ - extremely likely) were entered into a 3 (lineup medium) x 2 (witness confidence) x 2 (viewing conditions) analysis of variance (ANOVA). There was a main effect of viewing conditions, $F(1,394)=19.78, p<.01, \eta_{p}{ }^{2}=.05$, with participants more likely to rate the suspect as guilty when encoding conditions were described as well-lit than when they were described as dark. There was also a main effect of lineup medium, $F(2,394)=3.08, p=.047, \eta_{p}^{2}$ $=.02$. Post hoc tests indicated that participants were more likely to rate the suspect as guilty when the witness had made an identification from a video lineup than from a photo lineup, $p=$ $.01, d=0.31$ [95\% CI: 0.07, 0.55]. No other comparisons differed significantly, $p \mathrm{~s}>.14$. Descriptive data are presented in Table 1.

Participants made a dichotomous decision about suspect guilt (guilty/not guilty). A 2 (guilt) x 3 (medium) x 2 (viewing conditions) x 2 (certainty) hierarchical loglinear analysis revealed no interactions, $p \mathrm{~s}>.47$.

Participants then rated their confidence in their dichotomous guilt decision (from 0-100). We conducted two ANOVAs, one for participants who rated the suspect as guilty and one for those who rated the suspect as not guilty. There were no significant differences $(F \mathrm{~s}<1.6, p \mathrm{~s}>$ $.24)$. 
Table 1. Means (standard deviations) of participant guilt ratings (0 to 100).

\begin{tabular}{lllll}
\hline & \multicolumn{2}{c}{$70 \%$ Confidence } & \multicolumn{2}{c}{ 90\% Confidence } \\
View & Well-lit & Dark & Well-lit & Dark \\
\hline & & & & \\
Photo & $57.03(18.62)$ & $47.41(23.22)$ & $61.47(18.58)$ & $55.00(25.64)$ \\
Live & $63.51(16.62)$ & $54.39(19.36)$ & $65.63(20.11)$ & $47.03(24.40)$ \\
Video & $61.06(16.48)$ & $55.06(17.29)$ & $65.56(17.47)$ & $62.97(16.26)$ \\
\hline
\end{tabular}

Finally, participants were asked two open-ended questions about concerns related to the witness' identification and what may have enhanced confidence in the witness' identification. The most frequent concerns described about the identification were: general unreliability (42.6\%), lighting (34.5\%), witness confidence (29.1\%), witness believability (20.4\%), and lineup medium (7.1\%). The most common suggestions for what may have increased confidence in the identification were comments related to: more specific descriptions/more emotional witness (35.5\%), more favorable witnessing conditions (31.5\%), witness confidence (21.4\%), lighting $(16.3 \%)$, and lineup medium $(7.6 \%){ }^{1}$

Lineup beliefs. After responding to questions about the witness depicted in the video, participants provided general impressions of eyewitness identification lineup procedures. Participants were asked to indicate which of six lineup presentation methods (see Figure 1) would be preferable for making their own identification, as well as which method would lead to the most accurate identifications by another person. Some participants were excluded from the following analyses for selecting multiple mediums as preferable, resulting in a sample of 374 .

\footnotetext{
${ }^{1}$ Note. Percentages sum to more than $100 \%$ because participants could provide multiple responses.
} 
For the question about their own identification, a 2 (simultaneous vs sequential) x 3 (live vs. photo vs. video) hierarchical loglinear analysis revealed a significant two-way effect, $\chi^{2}(2)=$ $31.11, p<.001$. Partial associations indicated a significant effect for medium, $\chi^{2}(2)=381.49, p<$ .001 , and a significant effect for presentation, $\chi^{2}(1)=3.87, p=.049$. Live lineups $(82 \%)$ were preferred over both photo lineups $(6 \%), z=32.42, p<.001, O R=72.00$ [95\% CI: 43.47 , 119.24], and video lineups (12\%), $z=26.49, p<.001, O R=32.09$ [95\% CI: 21.39, 48.13], and simultaneous lineups (55\%) were preferred over sequential lineups (45\%), OR $=1.50$ [95\% CI: $1.13,2.01]$. The interaction indicated that the preference for simultaneous lineups only applied to the live medium. For the video medium, sequential was preferred. As can be seen in Figure 1, a very similar pattern of responses was observed for the question about which method would work best for someone else.

Figure 1. Proportion of participants rating each lineup medium as best for identifying a perpetrator (own identification and identifications by others)

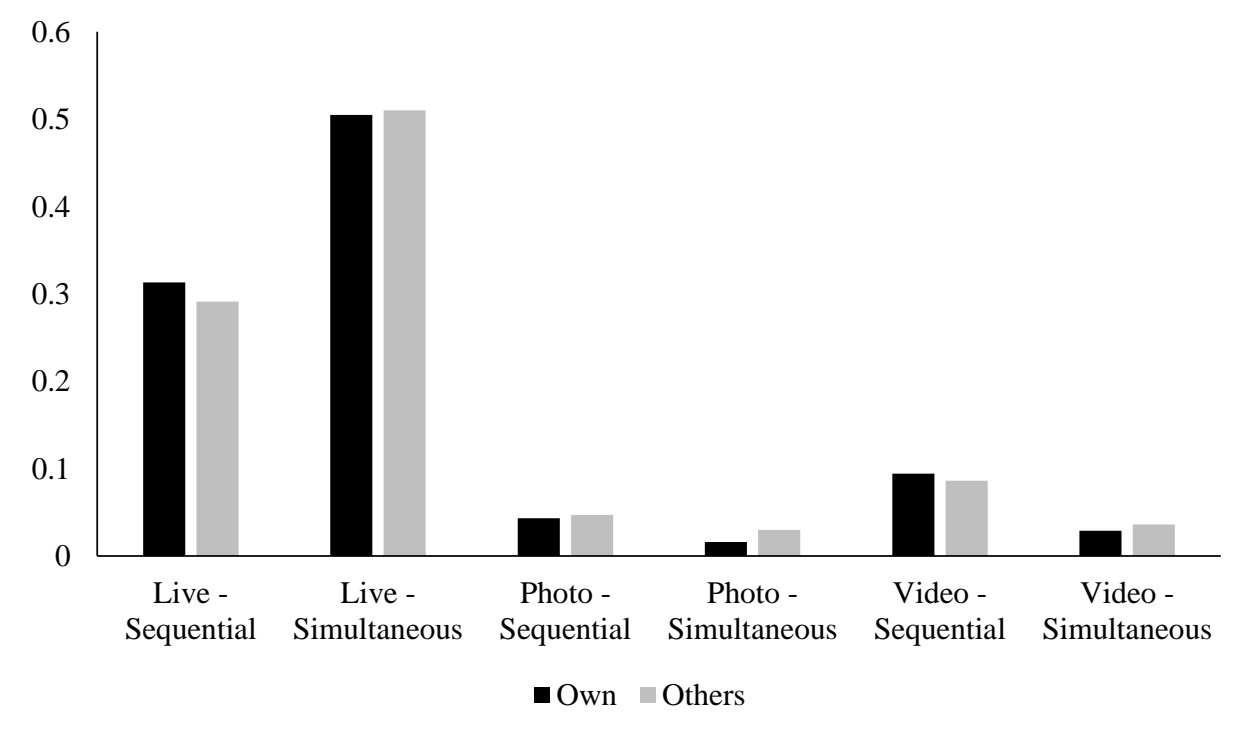


Participants then provided narrative explanations for their ratings. The most common reasons provided for preferring a particular lineup medium were: greater accuracy (54.2\%), live is more realistic (34.0\%), additional behavioral information provided (20.2\%), additional physical information provided (19.7\%), and photos can be examined more closely $(3.7 \%)$.

Finally, participants rated how confident they would be in making an identification from each lineup medium option, given good viewing conditions. A 2 (simultaneous vs sequential) x 3 (live vs. photo vs. video) repeated-measures ANOVA was performed and, due to Mauchley's test indicating that the assumption of sphericity was violated for tests involving the medium, $\chi^{2}(2)=$ $16.11, p<.001$, we applied the Greenhouse Geisser correction to the degrees of freedom for all tests involving the medium. The ANOVA revealed a main effect of medium, $F(2,774)=172.93$, $p<.001, \eta_{p}{ }^{2}=.309$, no main effect of presentation $F(1,387)=0.88, p=.348, \eta_{p}{ }^{2}=.002$, and a significant interaction, $F(2,774)=20.14, p<.001, \eta_{p}{ }^{2}=.049$. The main effect was again indicative of a preference for live lineups $(M=74.32, S D=15.97)$ over photo lineups $(M=$ 59.36, $S D=17.61)$ and video lineups $(M=61.54, S D=18.56)$. The interaction indicated higher ratings for simultaneous with live (simultaneous: $M=75.85, S D=19.75$; sequential: $M=72.48$, $S D=20.12$ ) and photo (simultaneous: $M=60.26, S D=20.38$; sequential: $M=58.23, S D=$ 20.56), versus higher ratings for sequential with video (simultaneous: $M=60.19, S D=21.63$; sequential: $M=62.87, S D=20.99)$.

\section{Discussion}

There is distinct international division in the perception of lineup medium best practices, as reflected in policy. Yet, there is little empirical evidence to guide policy development. Advancing our understanding of lineup identification accuracy as a function of lineup medium is a critical area for further inquiry, but it is also important to understand how potential triers of fact 
perceive evidence elicited by each medium. The clearest finding in the present study is that when participants were asked to directly compare lineup mediums, there was a strong preference for live lineups, thus supporting the existence of a live superiority hypothesis in potential witnesses and triers of fact.

\section{Evidence for a live superiority hypothesis}

The strong preference for live lineups when all mediums were directly compared provides the first evidence for a live superiority hypothesis among potential witnesses and triers of fact. Live lineups are associated with lower choosing rates, which may result from a more strict decision criterion. If witnesses and triers of fact believe that making an accurate identification is more likely from a live lineup relative to alternative options, they may have higher expectations for both themselves and others making such identifications. The demonstration that undergraduate students believe that live lineups are superior in the present research is an important step in developing a theoretical understanding of the medium's effect on eyewitness identification decisions. If the live superiority hypothesis is also prevalent among individuals in the justice system, this could also have applied implications. For instance, one can imagine an investigator may ask a witness to make an initial identification from a photo lineup, but subsequently assemble a live lineup to allow the witness to make an identification from a more desirable medium.

Why might live lineups be preferred over other mediums? As discussed above, there may be an intuitive belief that live lineups provide the most information and that more information is likely to lead to more accurate identifications. Indeed, in response to open-ended questions, participants reported believing that both live and video lineups were more realistic than photo lineups and that they provide behavioral and physical information beyond what is available in 
photo lineups. Implicit in these reports is a belief that this additional information would contribute to more accurate identifications - a supposition that may not always be true (see Fitzgerald et al., 2018 for review). There is some indication that additional physical cues might be able to enhance the ability to match images (Rice, Phillips, Natu, An, \& O'Toole, 2013), but the link has not yet been made to eyewitness identification accuracy.

\section{Evaluating lineup medium in isolation}

The preference for video over photo lineups was the only significant difference related to lineup medium that was observed in the experimental manipulations - and it was a small effect. There are several possibilities for why lineup medium did not have a substantive effect in the experiment. First, our manipulation of lineup medium may not have been especially salient to witnesses, particularly when other components of the witness' testimony were more intuitively

influential (e.g., lighting). When participants were asked to report concerns with the eyewitness identification depicted in the video, lineup medium was only rarely raised (7.1\%), suggesting that it may not be a primary consideration for participants unless consciously directed to attend to the medium. Further, when we asked participants to report which lineup medium had been used to elicit the identification, only $80 \%$ of participants in both the live $(80.7 \%)$ and photo (80.3\%) identification conditions were able to respond correctly, and $48.5 \%$ of participants in the video condition were able to correctly report the lineup medium used. This much lower correct response rate for video lineups may relate to the lack of familiarity with video lineups.

We also considered the possibility that our focus exclusively on sequential lineups in the experimental conditions may have influenced the preference for video lineups. For example, if video lineups were strongly preferred when presented sequentially (compared to simultaneously), our focus on sequential lineups may explain why video lineups were preferred 
in the experimental conditions, whereas live lineups were preferred in the general preferences questions. However, simultaneous and sequential lineups were rated very similarly in the current research and, if anything, the belief in live lineup superiority was slightly stronger in sequential (versus simultaneous) lineups.

\section{Relations to evidence strength}

Lineup medium did not interact with manipulations of evidence strength (witness confidence and viewing conditions). We had anticipated that weak evidence strength may be compensated for with what was perceived as a 'strong' medium, but this was clearly not the case. Although mock jurors have previously been found to be relatively insensitive to viewing conditions (e.g., Cutler, Penrod, \& Stuve, 1988), we observed overall effects of lighting on guilt ratings. However, the lack of interaction with lineup medium suggests that the influence of lineup medium may be evaluated independently from evidence strength. It is also possible that our manipulations of evidence strength were simply not strong enough to interact with medium (i.e., weak evidence was not 'weak enough').

As this area of research moves forward, an important contextual consideration is ecological validity in the use of mock crimes, mock witnesses, and mock jurors. Though such methods allow for the isolation of particular variables of interest and have been shown to not differ substantially from research on 'real' jury decision-making (see Bornstein, 1999), laboratory materials are unlikely to capture the important nuances and influences that will be present in real cases involving eyewitness identification. Thus, an important next step will be to continue to explore live superiority beliefs in multiple contexts.

A final point of interest is that eyewitness testimony is among the most influential forms of evidence (Semmler, Brewer, \& Douglass, 2011) and the persuasiveness of inaccurate 
eyewitness identifications has contributed disproportionally to wrongful convictions in DNA exoneration cases (www.innocenceproject.org). Most of the research conducted on the persuasiveness of eyewitness evidence, however, has focused on photo lineup identifications. Thus, it is possible that estimates of the persuasiveness of eyewitness evidence that rely on prior research with photo lineups may underestimate the influence of eyewitness evidence gathered using a different medium.

\section{Conclusion}

Despite strong jurisdictional guidance regarding how eyewitness identification procedures should be administered, there is relatively little evidence to support one medium over another. The ultimate aim of eyewitness identification is to provide evidence that can be evaluated in judicial proceedings. Thus, the perception of the evidence is critical. If a particular lineup medium produces a higher rate of accurate identifications, we must also consider the potential cost to credibility if triers of fact cannot appropriately evaluate the evidence. Similarly, if evidence is weighted too heavily when obtained with one particular medium over another, awareness must be brought to the judiciary about inappropriate influence. The present data suggest that when lineup mediums are compared directly, the belief in the superiority of live lineups is strong among potential witnesses and triers of fact. 


\section{References}

Australian Law Reform Commission. (2006). Uniform Evidence Law (ALRC Report 102).

Retrieved from http://www.alrc.gov.au/sites/default/files/pdfs/publications/ALRC102.pdf

Bornstein, B. H. (1999). The ecological validity of jury simulations: Is the jury still out? Law and Human Behavior, 23, 75-91. DOI: 10.1023/A:1022326807441

Brewer, N., \& Burke, A. (2002). Effects of testimonial inconsistencies and eyewitness confidence on mock-juror judgments. Law and Human Behavior, 26, 353-364. DOI: 10.1023/A:1015380522722

Brewer, N., \& Palmer, M. A. (2010). Eyewitness identification tests. Legal and Criminological Psychology, 15, 77-96. DOI: 10.1348/135532509X414765

Clark, S. E., Moreland, M. B., \& Rush, R. A. (2015). Lineup composition and lineup fairness. In T. Valentine \& J. P. Davis (Eds.), Forensic facial identification: Theory and practice of identification from eyewitnesses, composites and CCTV (pp. 127-157). Winchester, UK: John Wiley and Sons.

Cutler, B. L., \& Fisher, R. P. (1990). Live lineups, videotaped lineups, and photoarrays. Forensic Reports, 3, 439-449.

Cutler, B. L., Penrod, S. D., \& Stuve, T. E. (1988). Jury decision making in eyewitness identification cases. Law and Human Behavior, 12, 41-55. DOI: 10.1007/BF01064273

Cutler, B. L., Berman, G. L., Penrod, S. D., \& Fisher, R. P. (1994). Conceptual, practical, and empirical issues associated with eyewitness identification test media. In D. F. Ross, J. D. Read, \& M. P. Toglia (Eds.), Adult eyewitness testimony: Current trends and developments (pp. 163-181). New York: Cambridge University Press. 
Dent, H. R., \& Stephenson, G. M. (1979). Identification evidence: Experimental investigations of factors affecting the reliability of juvenile and adult witnesses. In D. P. Farrington, K. Hawkins, \& S. M. Lloyd-Bostock (Eds.), Psychology, law and legal processes (pp. 195206). Atlantic Highlands, NJ: Humanities Press.

Fitzgerald, R. J., Price, H. L., \& Valentine, T. (2018). Eyewitness identification: Live, photo, and video lineups. Psychology, Public Policy and Law, 24, 307-325. DOI:

10.1037/law0000164

Home Office. (2017). Police and Criminal Evidence Act 1984 (PACE) Code D. Retrieved from https://www.gov.uk/government/publications/pace-code-d-2017

Kerstholt, J. H., Koster, E. R., \& van Amelsvoort, A. G. (2004). Eyewitnesses: A comparison of live, video, and photo line-ups. Journal of Police and Criminal Psychology, 19, 15-22. DOI: $10.1007 / \mathrm{BF} 02813869$

Peters, D. P. (1991). The influence of stress and arousal on the child witness. In J. Doris (Ed.) The suggestibility of children's recollections: Implications for eyewitness testimony (pp. 60-76). Washington: American Psychological Association.

Pike, G., Brace, N., \& Kynan, S. (2002). The visual identification of suspects: Procedures and practice. Home Office Briefing Note. London, UK: Home Office Research, Development and Statistics Directorate. Retrieved from http://citeseerx.ist.psu.edu/viewdoc/download;jsessionid=AC651534A1894C27AEBD75 ECE61AC6A3?doi=10.1.1.608.8076\&rep=rep1\&type=pdf

Rice, A., Phillips, P. J., Natu, V., An, X., \& O’Toole, A. J. (2013). Unaware person recognition from the body when face identification fails. Psychological Science, 24, 2235-2243. DOI: $10.1177 / 0956797613492986$ 
Rust, A., \& Tredoux, C. (1998). Identification parades: An empirical survey of legal recommendations and police practice in South Africa. South African Journal of Criminal Justice, 11, 196-212.

S v Moti. (1998). SACR 245 (SCA)

Semmler, C., Brewer, N., \& Douglass, A. B. (2011). Jurors believe eyewitnesses. In B. L. Cutler (Ed.), Conviction of the innocent: Lessons from psychological research (pp. 185-209). Washington, DC: APA Books.

Valentine, T., Harris, N., Piera, A. C., \& Darling, S. (2003). Are police video identifications fair to African-Caribbean suspects? Applied Cognitive Psychology, 17, 459-476. http://dx.doi.org/10.1002/acp.880

Valentine, T., \& Heaton, P. (1999). An evaluation of the fairness of police line-ups and video identifications. Applied Cognitive Psychology, 13, S59 -S72. DOI:10.1002/acp.679

Valentine, T., Hughes, C., \& Munro, R. (2009). Recent developments in eyewitness identification procedures in the United Kingdom. In R. Bull, T. Valentine, \& T. Williamson (Eds.), Handbook of psychology of investigative interviewing: Current developments and future directions (pp. 221-240). Chichester: Wiley.

Yarmey, A. D. (1986). Verbal, visual, and voice identification of a rape suspect under different levels of illumination. Journal of Applied Psychology, 71, 363-370. 